\title{
Evaluation of the Auto Surfacing methods to create a surface body of the mandible model
}

\author{
Pawel Turek ${ }^{1}$ \\ ${ }^{1}$ Faculty of Mechanical Engineering and Aeronautics, Rzeszów University of Technology, Rzeszów, Poland, e-mail: \\ pturek@prz.edu.pl
}

\begin{tabular}{l} 
Article Info \\
\hline Article history: \\
Received September 11, 2021 \\
Revised December 21, 2021 \\
Accepted December 25, 2021 \\
\hline
\end{tabular}

\section{Keywords:}

Reverse engineering, Anatomical structures, Auto surfacing, Accuracy, CAD modelling.

\section{Corresponding Author:}

Paweł Turek

Faculty of Mechanical Engineering and Aeronautics, Rzeszów University of Technology, Rzeszów, Poland Email: pturek@prz.edu.pl

\begin{abstract}
Designing an anatomical structure for a surgical procedure is not a simple task. It is especially true of the craniofacial area, which consists of bone tissues with very complex geometry. CAD modelers need appropriate knowledge and skills in medicine and technical sciences to fully use the currently available tools in related processes with the reconstruction of the craniofacial areas. The presented preliminary studies are based on four patients treated at the Department of Maxillofacial Surgery. The segmentation process of the mandible model was performed in the ITK SNAP software. The process of generating surface body models was performed in the Auto Surfacing module in Geomagic software using two different methods: organic and mechanical. Then compare both methods for the accuracy of generating a CAD model of the mandible based on a triangle mesh structure in the Focus Inspection and the GOM Inspect software.
\end{abstract}

Copyright (C) 2021 Regional Association for Security and crisis management and European centre for operational research. All rights reserved.

\section{Introduction}

Reverse engineering is a process that makes it possible to reconstruct the geometry of an existing object (Raja and Fernandes (2007), Bagci (2009)). It covers activities related to data acquisition, reconstruction of the geometry of measured objects, and the transformation of the obtained data into a form that allows its use in CAD systems. When a physical object has a complex shape, the reverse engineering process is often the only way to create a digital model (Turek (2019), Ciocca et al. (2012)). The process of reverse engineering is, therefore, very complex. In order to correctly reconstruct the geometry of the object, the CAD modeler needs to have skills and knowledge about the measurement technique (Raja and Fernandes (2007)), data processing (Urbanic et al. (2008)), and manufacture technology (Boboulos (2010), Gibson et al. (2021)). Currently, reverse engineering is used in many areas, including the automotive (Marchetti and Stabili (2018)), and aviation industry (Kumar et al. (2013)). It is also very often used in medicine - e.g., reconstructing the geometry of anatomical structures (Stojkovic et al. (2018), Turek (2019)) and design implants (Milovanović et al. (2020)), surgical guide (Liu et al. (2014), Orabona et al. (2018)). The made three-dimensional models of bone structures allow for:

- $\quad$ better preparation of the doctor for the procedure;

- $\quad$ increasing the precision of the operation;

- $\quad$ selection of appropriate surgical instruments;

- consulting the case more carefully with other doctors before starting the procedure;

- $\quad$ presenting the patient in more detail with the scope of the procedure and discussing its course;

- $\quad$ shortening of the time of surgery (general anesthesia);

- reduction of blood loss during surgery;

- minimizing intraoperative complications. 
When designing anatomical structures, the first step is to obtain volumetric data. It can be done through the use of tomographic systems (Cohen et al. (2009)), magnetic resonance imaging (Huotilainen et al. (2014)) and ultrasonography (Vaezi et al. (2012)). Then the obtained data is transformed into a three-dimensional model. Various types of software are used for this purpose. The commercial program Mimics (Figliuzzi et al. (2012)), 3D Doctor (Teeter et al. 2015)), Amira (Maret et al. (2012)) and Maxilin (Olszewski et al. (2014)) appears most often in the literature. It is very important to research the accuracy of the geometry reconstruction and the manufacture of the physical model (Budzik and Turek (2018) and Turek and Budzik (2021)). Currently, at the stage of reconstructing the model geometry, research is being carried out to improve the accuracy of segmentation of the anatomical structures (van Eijnatten et al. (2017) and (2018)), triangulation algorithms (Manmadhachary et al. (2016)) and CAD modeling (Yoo (2011)). In the case of manufacturing models of anatomical structures using additive techniques, it is necessary to check the model saved in the STL format for structure errors (Manmadhachary (2016)). Considering machining methods, it is necessary to generate a CAD model based on the STL model accurately. Creating an accurately generated surface body based on a triangle mesh structure can significantly reduce the preparation time of the machining process (Budzik et al. (2015)).

The literature analysis shows the increasing use of CAD/CAM/RP systems in medicine. Knowing of designing anatomical models can help a lot in the future in the controlled preparation of templates and surgical guides in terms of the accuracy expected during the procedures. They can also be supported in the process of carrying out, among other things, procedures to restore the continuity of the mandible geometry, as well as treatment of other diseases of the skeletal system.

\section{Materials and methods}

Models of the four mandibles were obtained from the Siemens Somatom Sensation Open 40 tomograph. Digital Imaging and Communications in Medicine (DICOM) data was provided from the Provincial Clinical Hospital no. 1. Frederic Chopin in Rzeszów. The traditional scanning protocol for the craniofacial scan was used (Table 1).

Table 1. The scanning protocol.

\begin{tabular}{cc}
\hline Name of parameters & Value of parameters \\
\hline Tube voltage & $120 \mathrm{kV}$ \\
\hline Tube current-time product & $115 \mathrm{mAs}$ \\
\hline Acquisition & $40 \times 0,6 \mathrm{~mm}$ \\
\hline Slice collimation & $0,6 \mathrm{~mm}$ \\
\hline Kernel & $\mathrm{H} 60 \mathrm{~s}$ \\
\hline Matrix size & $512 \times 512$ \\
\hline Pixel size & $0,4 \times 0,4 \mathrm{~mm}$ \\
\hline
\end{tabular}

Based on the prepared data, the segmentation process of the mandible model was performed in the ITK SNAP software. The value of $200 \mathrm{HU}$ was selected as the lower threshold value. In order to visualize the 3D model of the mandible, the isosurface method was used, which is part of the surface rendering methods. This method is based on the marching cubes algorithm. This algorithm proceeds through the scalar field, taking eight neighbor locations at a time (thus forming an imaginary cube), then determining the polygon(s) needed to represent the part of the isosurface that passes through this cube. The individual polygons are then fused into the desired surface. It is done by creating an index to a recalculated array of 256 possible polygon configurations within the cube by treating each of the eight scalar values as a bit in an 8-bit integer. If the scalar's value is higher than the iso-value (i.e., inside the surface), then the appropriate bit is set to one, while it is lower (outside), it is set to zero. The final value, after all, eight scalars are checked, is the actual index to the polygon indices array. Finally, each vertex of the generated polygons is placed on the appropriate position along the cube's edge by linearly interpolating the two scalar values connected by that edge. This algorithm guarantees that the resulting surfaces are free from cracks and holes, that no triangles (single surface built on three nearest points) intersect, and that all regions assigned to different materials are well separated from each other. The final model of the mandible has been saved as an STL(STereoLitography) file (Fig.1) and next imported into the Geomagic software. The Geomagic software gives many tools for editing and manipulating the data obtained during the digitization process. The main task of this software is to convert the point cloud to a triangle mesh or free surfaces described by NURBS (Non-Uniform Rational B-Spline) curves while trying to obtain the best possible accuracy of the physical model. Due to inaccuracies occurring during the measurements, the fundamental errors in the triangle mesh were verified: 
- inverted normal vectors;

- $\quad$ gaps between the triangles describing the surface;

- $\quad$ surface distortions;

- loss of whole surfaces or their fragments;

- overlapping triangles;

- $\quad$ assigning a single edge to more than one triangle.

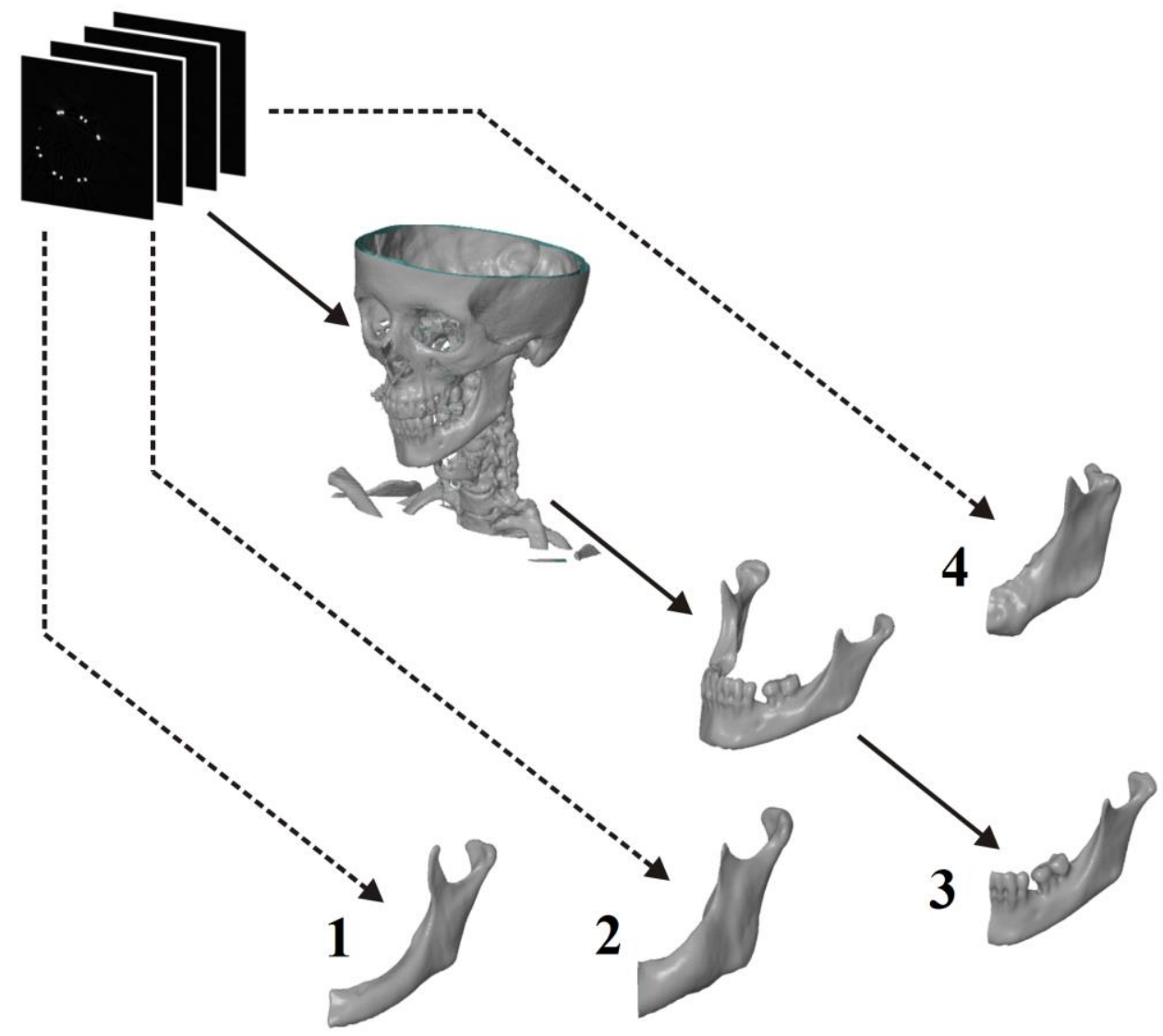

Figure 1. The models representing a mandible geometry (triangle mesh)

In the test results, no errors were found in the structure of the mesh. The final part of preparing readymade models consisted in defining the cutting plane against which ready-made models of the mandible sections were obtained. The process of generating surface models was performed in the Auto Surfacing module. Auto surfacing creates a surface body that can envelop the entire geometric shape of a target mesh. It provides two different methods:

- Organic: Create a surface body from a target mesh by projecting an evenly distributed curve network (Fig.2);

- Mechanical: Create a surface body from a target mesh by constructing a curve network following the features (Fig.3).

The entire process of generating the mandible sections' surface model consisted of creating spline approximation curves on the obtained triangle mesh using the organic and then the mechanical method. As a result, an edge model was obtained, then used to distribute the panels over its entire surface. The final step was to combine all the created surfaces into one coherent whole. The process uses default settings for the accuracy of fitting the surface slices to the triangle mesh. 


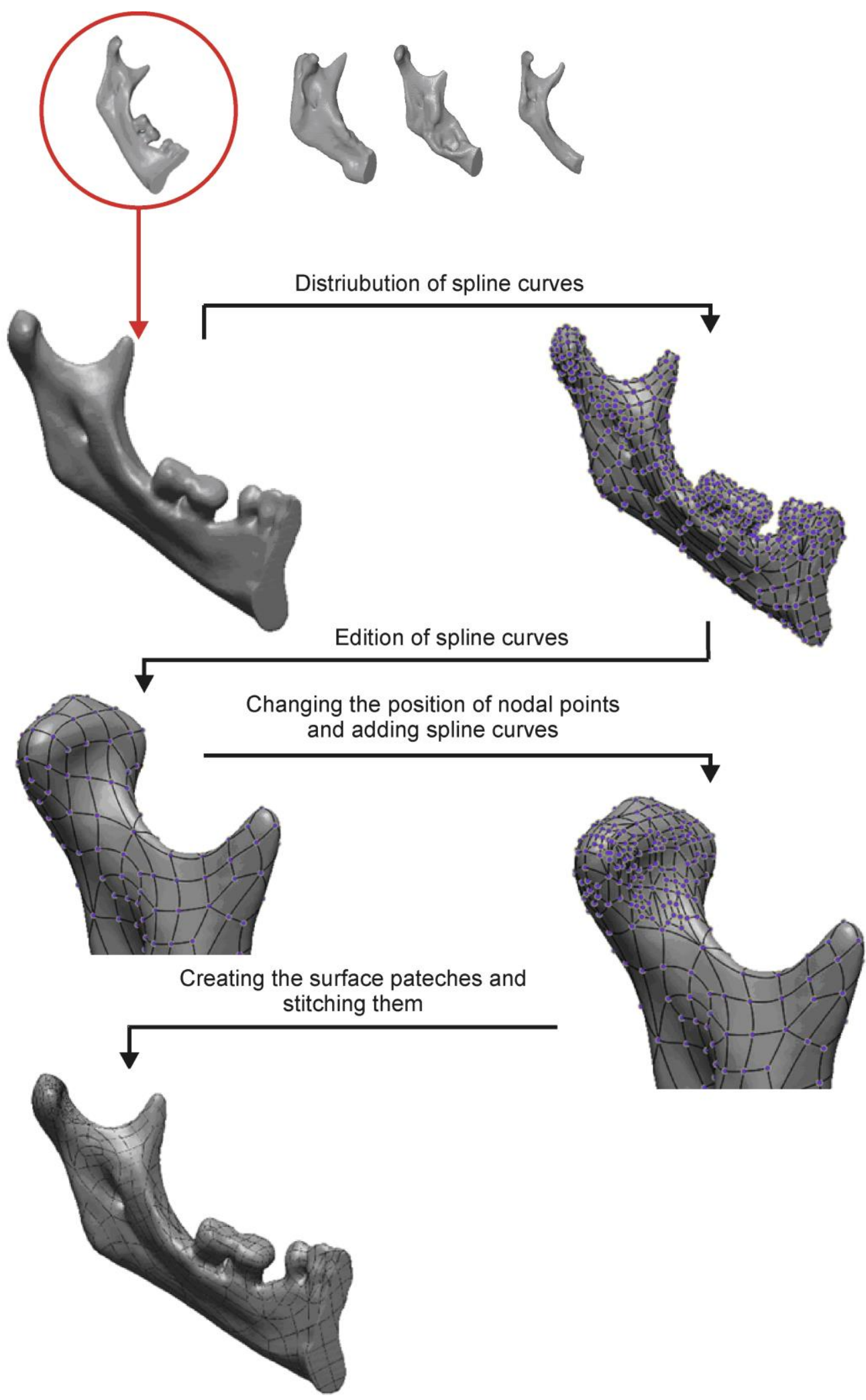

Figure 2. The scheme of designing a mandible CAD model - organic method 


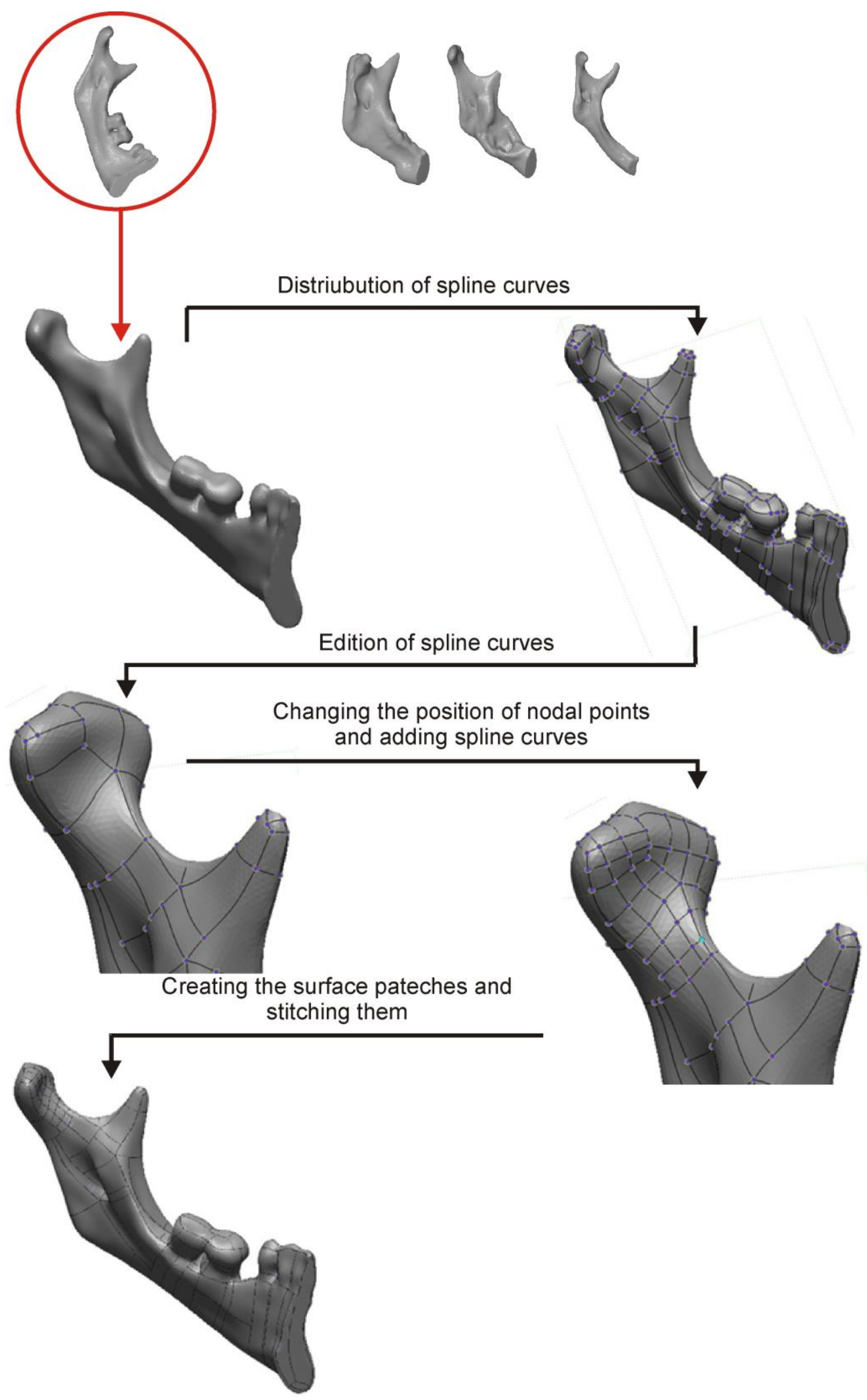

Figure 3. The scheme of designing a mandible CAD model - mechanical method 


\section{Results}

As part of the presented research, the CAD modeling methods were assessed to match the surface patches to the triangle mesh structure. The process of verifying the procedures was carried out on the Focus Inspection and GOM Inspect software. The fitting of the nominal model (triangle mesh) and the reference model (CAD model) was performed using the best fit method with an accuracy of $0.001 \mathrm{~mm}$.

a)

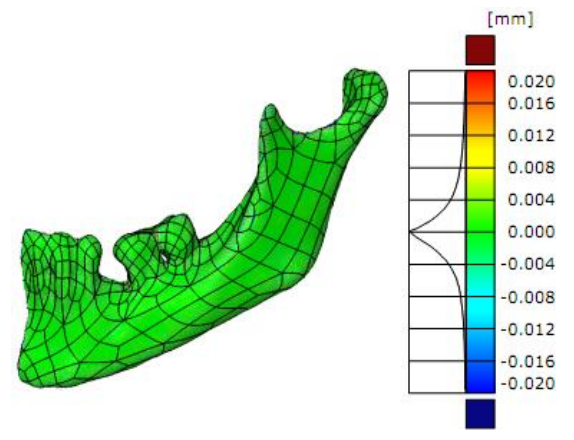

c)

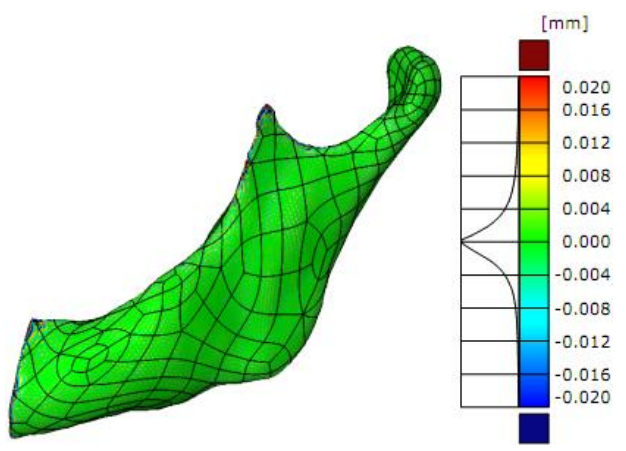

e)

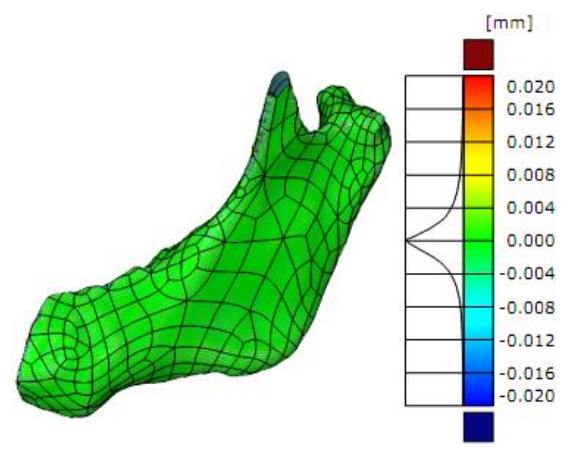

g)

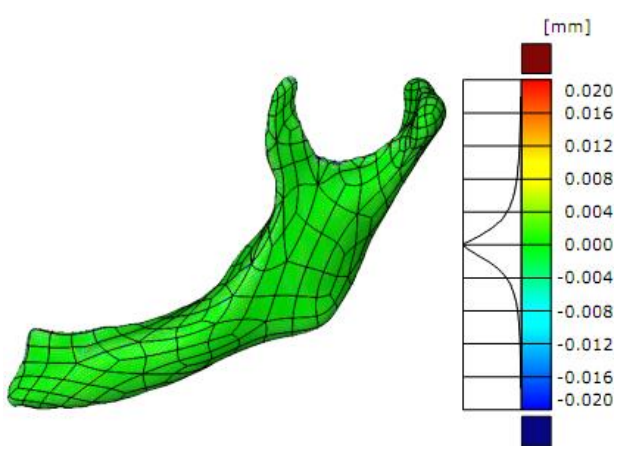

b)

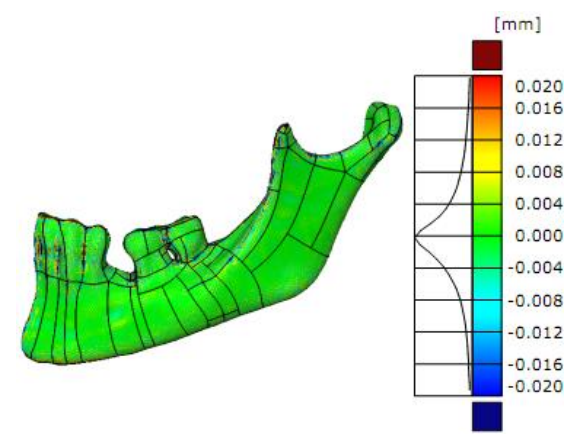

d)

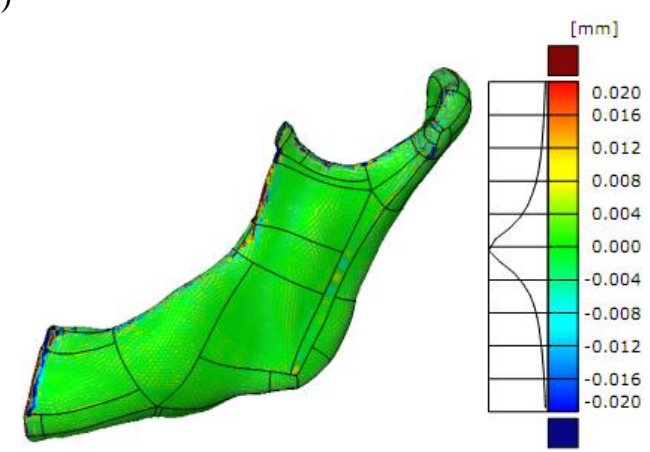

f)

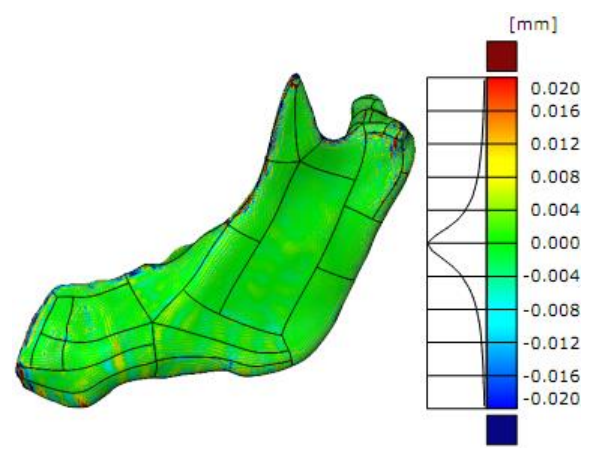

h)

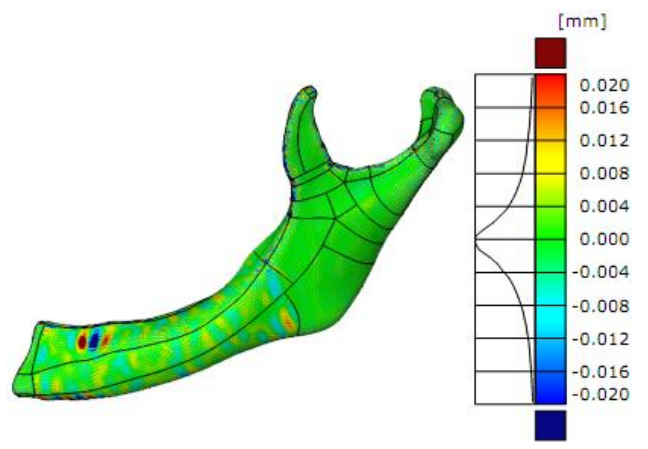

Figure 4. CAD model generation accuracy report: (a) model 1 - organic method; (b) ) model 1 mechanical method; (c) ) model 2 - organic method; (d) model 2 - mechanical method; (e) model 
3 - organic method; (f) model 3 - mechanical method; (g) model 4 - organic method; (h) ) model 4 - mechanical method;

\section{Discussion}

Two methods were used in the 3D modeling process - CAD of the mandible models - Organic and Mechanical. Both methods allowed for the creation of approximation splain curves on the obtained triangle mesh. As a result, an edge model was created on which the surface patches were spread. The great advantage of the methods used is the ability to control the course of the generated curves (Fig.5). The difference between them is how they are generated.

a)

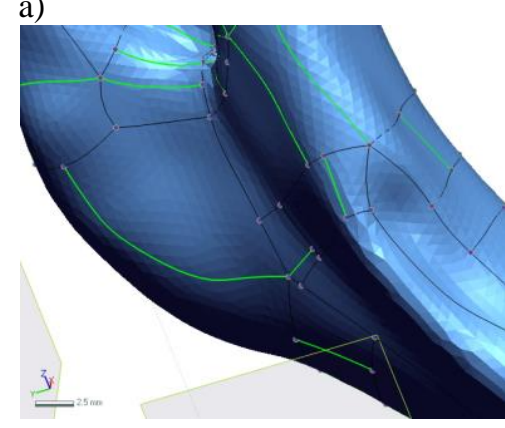

b)

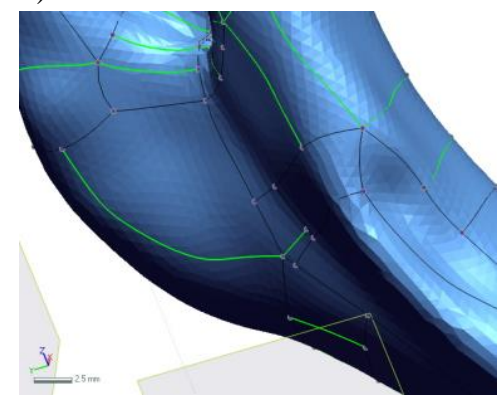

c)

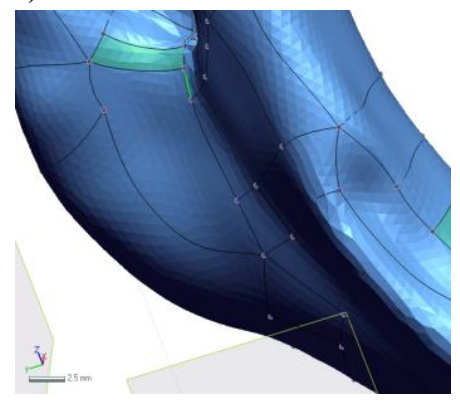

Figure 5. Process of editing curves: (a) View before editing; (b) After removing curves; (c) after joining

In the case of the first method, more regular shapes of the surface patches were obtained. The second method is more focused on minimizing the number of patches on the surface of the 3D CAD model. In the modeling process, care was taken in particular about the most accurate representations of the mandible's lateral surface and the condylar process's geometry. These areas are mainly used in designing implants and tools to improve the course of surgery and rehabilitation of the patient in the mandibular area. Problems were encountered with method one with frequent non-closing of surface patches and longer data processing time. In addition, reports assessing the accuracy of matching the patches to the surface of the models mainly indicate an increase in the standard deviation in the first method. The results show that in the case of modeling the surface of anatomical objects, the increase in the number of surface patches does not necessarily positively affect the accuracy of the surface model generation. Both methods have a problem with mapping the sharp edges of models. Considering the two procedures, the Mechanical method works best in modeling the lateral sections of the mandible.

\section{Conclusion}

Designing an anatomical structure model for surgery is not a simple task. It is especially true of the craniofacial area, which consists of bone tissues with very complex geometry. CAD modelers need appropriate knowledge and skills in medicine and technical sciences to fully use the currently available tools in related processes to reconstruct the craniofacial areas. It is especially true of the lower jaw, which is the only movable bone in this area. The preliminary research results on the accuracy of generating a CAD model based on a triangle mesh is a starting point for further, which will focus on developing a methodology for designing surgical templates and implants within the mandibular area.

Acknowledgement: The author would like to thank the Regional Clinical Hospital No. 1 in Rzeszow for DICOM data of 4 patients obtained on the Siemens Somatom Sensation Open 40 scanner installed in this clinic. 


\section{References}

Bagci, E. (2009). Reverse engineering applications for recovery of broken or worn parts and re-manufacturing: Three case studies. Advances in Engineering Software, 40, 407-418.

Boboulos, M. A. (2010). CAD-CAM \& rapid prototyping application evaluation. Bookboon.

Budzik, G., Burek, J., Dziubek, T., Gdula, M., Płodzień, M., \& Turek, P. (2015). The analysis of accuracy zygomatic bone model manufactured by 5-axis HSC 55 linear. Mechanik, 88.

Budzik, G., \& Turek, P. (2018). Improved accuracy of mandible geometry reconstruction at the stage of data processing and modeling. Australasian physical \& engineering sciences in medicine, 41, 687-695.

Ciocca, L., Mazzoni, S., Fantini, M., Persiani, F., Baldissara, P., Marchetti, C., \& Scotti, R. (2012). A CAD/CAM-prototyped anatomical condylar prosthesis connected to a custom-made bone plate to support a fibula free flap. Medical \& biological engineering \& computing, 50, 743-749.

Cohen, A., Laviv, A., Berman, P., Nashef, R., \& Abu-Tair, J. (2009). Mandibular reconstruction using stereolithographic 3-dimensional printing modeling technology. Oral Surgery, Oral Medicine, Oral Pathology, Oral Radiology, and Endodontology, 108, 661-666.

Figliuzzi, M., Mangano, F., \& Mangano, C. (2012). A novel root analogue dental implant using CT scan and CAD/CAM: selective laser melting technology. International journal of oral and maxillofacial surgery, 41, $858-862$.

Gibson, I., Rosen, D. W., Stucker, B., \& Khorasani, M. (2021). Additive manufacturing technologies (Vol. 17). Cham, Switzerland: Springer.

Huotilainen, E., Paloheimo, M., Salmi, M., Paloheimo, K. S., Björkstrand, R., Tuomi, J., \& Mäkitie, A. (2014). Imaging requirements for medical applications of additive manufacturing. Acta Radiologica, 55, 78-85.

Kumar, A., Jain, P. K., \& Pathak, P. M. (2013). Reverse engineering in product manufacturing: an overview. DAAAM international scientific book, 39, 665-678.

Liu, Y. F., Xu, L. W., Zhu, H. Y., \& Liu, S. S. Y. (2014). Technical procedures for template-guided surgery for mandibular reconstruction based on digital design and manufacturing. Biomedical engineering online, 13, $1-15$.

Manmadhachary, A., Kumar, R., \& Krishnanand, L. (2016). Improve the accuracy, surface smoothing and material adaption in STL file for RP medical models. Journal of Manufacturing Processes, 21, 46-55.

Marchetti, M., \& Stabili, D. (2018). READ: Reverse engineering of automotive data frames. IEEE Transactions on Information Forensics and Security, 14, 1083-1097.

Maret, D., Telmon, N., Peters, O. A., Lepage, B., Treil, J., Inglèse, J. M., \& Sixou, M. (2012). Effect of voxel size on the accuracy of 3D reconstructions with cone beam CT. Dentomaxillofacial Radiology, 41, 649-655.

Milovanović, J., Stojković, M., Trifunović, M., \& Vitković, N. (2020). Review of bone scaffold design concepts and design methods. Facta Universitatis, Series: Mechanical Engineering. DOI: 10.22190/FUME200328038M

Olszewski, R., Szymor, P., \& Kozakiewicz, M. (2014). Accuracy of three-dimensional, paper-based models generated using a low-cost, three-dimensional printer. Journal of Cranio-Maxillofacial Surgery, 42, 1847-1852.

Orabona, G. D. A., Abbate, V., Maglitto, F., Bonavolontà, P., Salzano, G., Romano, A., \& Califano, L. (2018). Low-cost, self-made CAD/CAM-guiding system for mandibular reconstruction. Surgical oncology, 27, 200207.

Raja, V., \& Fernandes, K. J. (2007). Reverse engineering: an industrial perspective. Springer Science \& Business Media.

Stojkovic, M., Veselinovic, M., Vitkovic, N., Marinkovic, D., Trajanovic, M., Arsic, S., \& Mitkovic, M. (2018). Reverse modelling of human long bones using T-splines-case of tibia. Tehnicki Vjesnik, 25, 17531760 . 
Teeter, M. G., Kopacz, A. J., Nikolov, H. N., \& Holdsworth, D. W. (2015). Metrology test object for dimensional verification in additive manufacturing of metals for biomedical applications. Proceedings of the Institution of Mechanical Engineers, Part H: Journal of Engineering in Medicine, 229, 20-27.

Turek, P. (2019). Automating the process of designing and manufacturing polymeric models of anatomical structures of mandible with Industry 4.0 convention. Polimery, 64, 522-529.

Turek, P., \& Budzik, G. (2021). Estimating the Accuracy of Mandible Anatomical Models Manufactured Using Material Extrusion Methods. Polymers, 13, 2271.

Urbanic, R. J., El Maraghy, H. A., \& El Maraghy, W. H. (2008). A reverse engineering methodology for rotary components from point cloud data. The International Journal of Advanced Manufacturing Technology, 37, 1146-1167.

Vaezi, M., Chua, C. K., \& Chou, S. M. (2012). Improving the process of making rapid prototyping models from medical ultrasound images. Rapid Prototyping Journal. 18, 287-298

Van Eijnatten, M., van Dijk, R., Dobbe, J., Streekstra, G., Koivisto, J., \& Wolff, J. (2018). CT image segmentation methods for bone used in medical additive manufacturing. Medical engineering \& physics, 51, 6-16.

van Eijnatten, M., Koivisto, J., Karhu, K., Forouzanfar, T., \& Wolff, J. (2017). The impact of manual threshold selection in medical additive manufacturing. International journal of computer assisted radiology and surgery, 12, 607-615.

Yoo, D. J. (2011). Three-dimensional surface reconstruction of human bone using a B-spline based interpolation approach. Computer-Aided Design, 43, 934-947. 